\title{
HospitalizaCiONES \\ Prevenibles Por condiciones \\ SENSIBLES AL CUIDADO AMBULATORIO \\ EN CINCO HOSPITALES DE LA RED \\ PÚBLICA DE BoGOTÁ 2006-2008
}

\section{Resuman}

Las condiciones sensibles al cuidado ambulatorio - CSCA-son indicadores de enfermedades para las cuales la Atención Primaria de Salud - APS- tiene capacidad potencial para evitar su hospitalización innecesaria. En el tamizaje de los egresos de cinco hospitales de la Red Pública de Bogotá se encontró que $22.5 \%$ de sus pacientes correspondieron a CSCA; $56 \%$ de los diagnósticos fueron de enfermedades infecciosas y parasitarias, $42 \%$ de enfermedades crónicas, $2 \%$ de otras enfermedades y $0.28 \%$ al cáncer de cuello uterino. Este estudio piloto es un inicio de la evaluación de la APS y su papel frente a la contención de los costos de la hospitalización.

\section{Palabras clave}

Cuidado ambulatorio Hospitalización Atención Primaria en Salud
Oswaldo Cáliz Peña*

\section{InTRODUCCí́n}

a reforma del sistema de salud en Colombia, puesta en marcha por la ley 100 de 1993, promueve un modelo de atención que se fundamenta sobre los servicios de atención primaria, que actúan como puertas de entrada a la atención especializada. La estrategia fundamental de este nivel es el cuidado ambulatorio de problemas de salud que son susceptibles de tratamiento con recursos de baja complejidad. De este modelo de atención se espera que una reducción de las hospitalizaciones potencialmente evitables por el cuidado ambulatorio, mejorara la cobertura, la calidad y los costos del sistema para la población.

Médico de la Universidad de Cartagena, especialista en Salud Pública de la Universidad de Antioquia y Magíster en Salud Pública de la Universidad de Antioquia. Docente en las universidades Jorge Tadeo Lozano, San Martín y la Santo Tomas de Bogotá y Tunja. 
Para evaluar el resultado de la estrategia descrita se ha desarrollado el indicador Condiciones Sensibles al Cuidado Ambulatorio (1) (CSCA), que sirve de medida directa de las hospitalizaciones potencialmente evitables e indirecta del volumen de actividad clínica que podría haber sido atendida o prevenida en la Atención Primaria (APS). Se considera que las CSCA identifican problemas de salud que corresponden a la Atención Primaria (esencialmente ambulatoria) porque podrían ser resueltos mediante algunas de las intervenciones propias de este nivel asistencial: prevención primaria, secundaria o terciaria (2). Estas intervenciones se dirigirán en algunos casos al mismo proceso que genera la hospitalización, como la diabetes o la hipertensión arterial esencial, mientras que en otros la actuación se dirigirá al precursor de la enfermedad que generará el ingreso, como es el caso de la sífilis congénita y las alteraciones nutricionales (3).

La utilización de este indicador fue introducida en los Estados Unidos para analizar el acceso de la población indigente a la atención médica (4). En la segunda mitad de los años noventa, este indicador fue propuesto por el Sistema Nacional de Salud Británico para el análisis de la calidad de la Atención Primaria. En la actualidad muchos países lo utilizan como indicador para monitorear la evolución y funcionamiento del sistema de salud.

\section{ABSTRACT}

Las condiciones sensibles al cuidado ambulatorio-CSCA-son indicadores de enfermedades para las cuales la Atención Primaria en Salud-APStiene capacidad potencial para evitar su hospitalización innecesaria. En el tamizaje de los egresos de cinco hospitales de la Red Pública de Bogotá se encontró que $22.5 \%$ de sus pacientes correspondieron a

CSCA; $56 \%$ de los diagnósticos fueron de enfermedades infecciosas y parasitarias, $42 \%$ de enfermedades crónicas, $2 \%$ de otras enfermedades y $0,28 \%$ al cáncer de cuello uterino. Este estudio piloto es un inicio de la evaluación de la APS y su papel frente a la contención de los costos de hospitalización.

\section{KIYY WORDS}

Cuidado ambulatorio

Hospitalización

Atención Primaria en Salud 
Varios autores describen el nivel de educación e ingresos de la población como inversamente proporcionales a las tasas de hospitalización por CSCA (5) (6). Otros sugieren que podrían evitarse mejorando la accesibilidad a los médicos de atención primaria porque hubiera posibilitado la detección de la enfermedad en un estadio temprano (7).

Los resultados de algunos estudios, sugieren que los pacientes sin seguro poseen índices más altos de hospitalización para condiciones que pueden ser tratadas, a menudo, fuera del hospital o evitado en su conjunto, dado que mejorando la accesibilidad a los servicios de atención primaria se hubiera detectado en una etapa menos avanzada de la enfermedad (8).

Otros estudios revelan que las bajas hospitalizaciones por CSCA están asociadas al acceso a una atención primaría eficaz y no tanto a los recursos profesionales disponibles en el área de salud (número y disponibilidad de médicos generales) (9) (10). Esta hipótesis fue contrastada también en un estudio comparativo entre Estados Unidos y España, donde se encontró que el índice de hospitalizaciones por CSCA eran más bajas en España que en Estados Unidos, lo cual fue asociado a un seguro universal en España que provee servicios de atención primaria sin pago directo (11).

Más específicamente, un grupo de autores establecen que las tasas de hospitalizaciones por CSCA están asociadas a la función del médico general como puerta de entrada al sistema (12) (13).

En este informe se presentan los resultados de un estudio piloto para analizar la aplicación del indicador CSCA en cinco hospitales de tercer nivel de atención de la red pública de Instituciones de Salud en Bogotá, como inicio de la evaluación de la Atención Primaria en Salud y su papel frente a la contención de los costos de la hospitalización. 


\section{Metodología}

\subsection{Tipo de estudio}

Estudio piloto observacional transversal de 263.917 egresos hospitalarios generados entre los años 2006 y 2008 en los hospitales Occidente de Kennedy, Simón Bolívar, La Victoria, Santa Clara y El Tunal.

\subsection{Unidad de análisis}

La unidad de análisis fue la hospitalización individual registrada en el egreso. Variables del estudio.

Frecuencia y porcentaje de hospitalización por CSCA y la edad de los pacientes clasificada en tres grupos: <de 15 años, de 15 a 64 y >65.

\subsection{Instrumento}

Se utilizó un listado de códigos de diagnóstico de CSCA seleccionado de la literatura adicionándoles algunos diagnósticos para Bogotá (Cáncer de cuello uterino, Sífilis congénita, Parasitosis intestinal, Enfermedad Diarreica Aguda, desnutrición, Anemia Ferropénica y Celulitis), y se agruparon en cuatro categorías: enfermedades crónicas, cáncer de cuello uterino, enfermedades infecciosas y parasitarias y otras condiciones, tabla 1.

A los hospitales del estudio se les solicitó el archivo de egresos en formato Excel para facilitar su tamizaje contra la lista de códigos seleccionados. Algunos hospitales no entregaron los archivos con las variables solicitadas, como edad y los días estancia, ninguno incluyó los costos de las hospitalizaciones. 
TABLA 1

Listado de códigos C|E-10 de CSCA adaptado para Bogotá

\begin{tabular}{|c|c|}
\hline CSCA & Códigos CIE-10 \\
\hline \multirow{2}{*}{$\begin{array}{l}\text { Enfermedades crónicas } \\
\text { Hipertensión arterial esencial }\end{array}$} & \\
\hline & $|10| 110-,\mid 119$ \\
\hline Infarto agudo del miocardio & I210-I219 \\
\hline Insuficiencia cardiaca & I500, I501, 1509 \\
\hline Enfermedad cerebrovascular & 1610-1629 \\
\hline Diabetes mellitus & E100-E109, E149 \\
\hline Enfermedad pulmonar obstructiva crónica & $\mathrm{J} 440-\mathrm{J} 449$ \\
\hline Asma & J450-J459, J46 \\
\hline Cáncer de cuello uterino & C530, C531, C538, C539 \\
\hline \multirow{2}{*}{$\begin{array}{l}\text { Enfermedades infecciosas } \\
\text { Bronquiolitis }\end{array}$} & \\
\hline & J210, J218, J219 \\
\hline Bronquitis aguda & $\mathrm{J} 200-\mathrm{J} 209$ \\
\hline Neumonía & $\begin{array}{l}\text { J120-J129, J13,J14, J150-J159, } \\
\text { J160, J168, J170-J173, J180-J189 }\end{array}$ \\
\hline Bronconeumonía & $\mathrm{J} 178$ \\
\hline Bronquitis aguda & $\mathrm{J} 200-\mathrm{J} 209$ \\
\hline Infección urinaria sitio no especificado & N390 \\
\hline Sífilis congénita & A500-A509 \\
\hline Tuberculosis pulmonar & A150-A159 \\
\hline Enfermedad diarreica aguda sin especificar & A09 \\
\hline Rinofaringitis aguda & $\mathrm{J} 00$ \\
\hline Otitis media no supurativa y no especificada & $\mathrm{H} 650-\mathrm{H} 659$ \\
\hline Celulitis & L030-L033, L038-L039 \\
\hline Parasitosis intestinal & A060-A069 \\
\hline \multirow{2}{*}{$\begin{array}{l}\text { Otras condiciones } \\
\text { Anemia ferropénica }\end{array}$} & \\
\hline & D500-D509 \\
\hline Desnutrición & E43, E440, E441, E40, \\
\hline Gastritis & K291-K299, K30 \\
\hline
\end{tabular}




\subsection{Las siguientes fueron las etapas metodológicas del estudio}

- Selección de las CSCA mediante la revisión bibliográfica de estudios similares.

- Obtención de los archivos de egresos (en formato de Excel) de los cinco hospitales seleccionados de tercer nivel de la Red Pública Hospitalaria de Bogotá.
- Tamizaje de los las bases de datos de los egresos de los cinco hospitales para identificar las CSCA, entre el 2006 y el 2008.

- Procesamiento y análisis los datos. No se calcularon tasas de hospitalización ya que en la población de referencia que sirven convergen varios hospitales de igual nivel de complejidad.

\section{Resultados}

Uas hospitalizaciones por CSCA representan en estos cinco hospitales $22.5 \%$ de sus egresos totales entre los años 2006 y 2008. Mientras el promedio porcentual del hospital Simón
Bolívar en el periodo del estudio fue $33.16 \%$, alcanzando la cifra de $45.09 \%$ en el 2008, a expensas de los egresos por EPOC y Neumonía; el de los otros cuatro hospitales fue $17.68 \%$, gráfica 1 .

GRÁFICA 1

Porcentaje de hospitalizaciones potencialmente evitables en cinco hospitales de la red pública de Bogotá

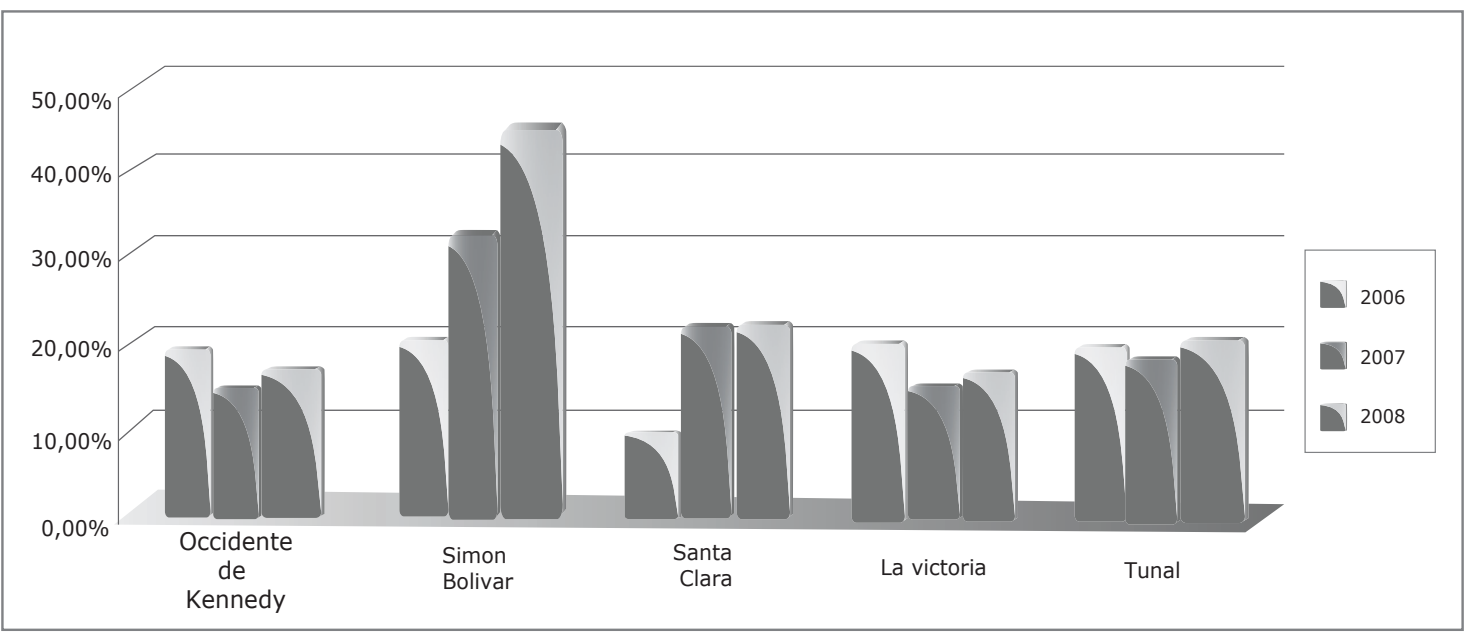

Fuente: RIPS de egresos hospitalarios 2006-2008 
Según las categorías en que se agruparon los diagnósticos de CSCA, se observó un predominio de las enfermedades infecciosas seguidas de las crónicas, gráfica 2.

\section{GRÁFICA 2}

Porcentaje de hospitalizaciones potencialmente evitables por grupos de causas en cinco hospitales

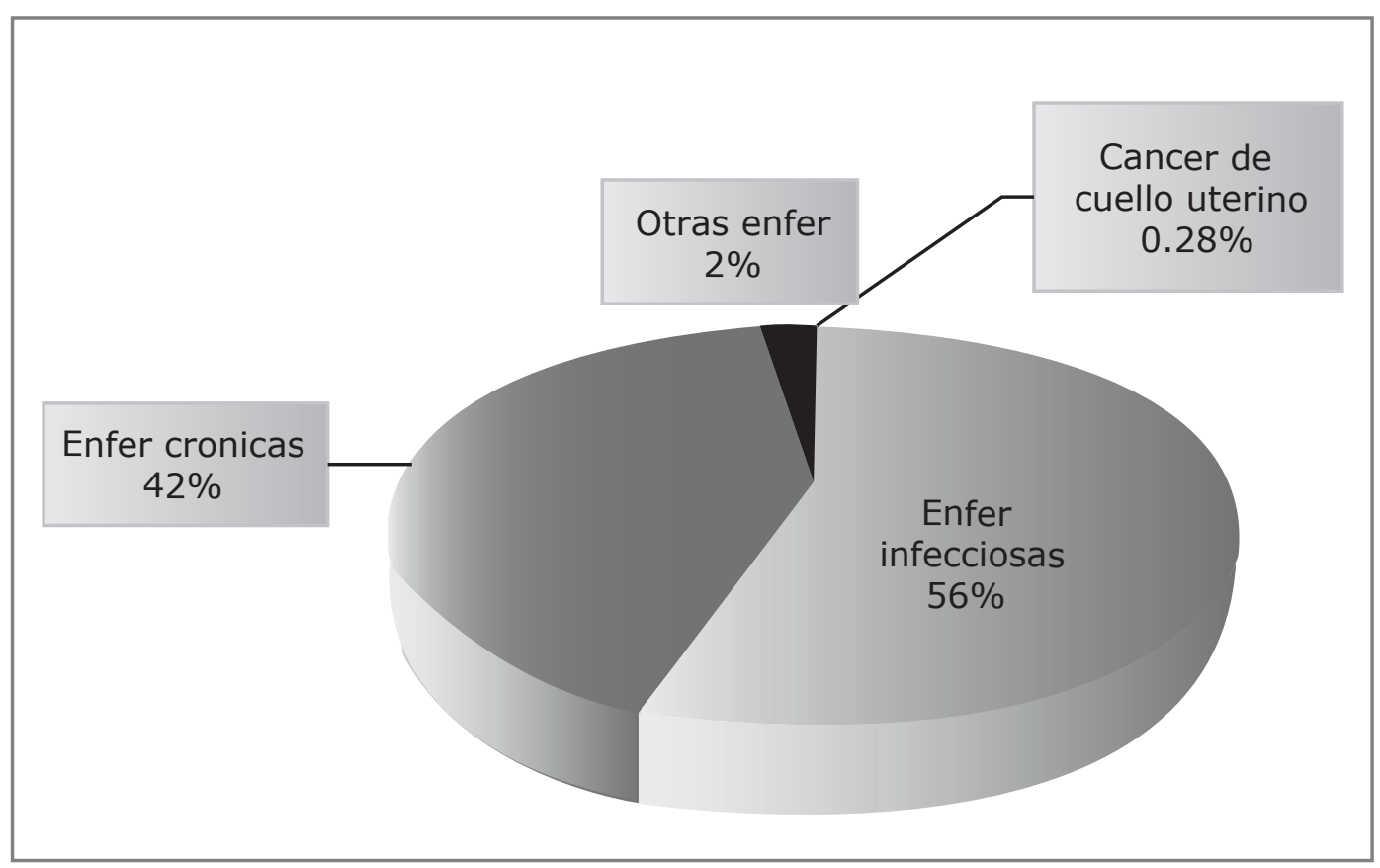

Fuente: RIPS de egresos hospitalarios 2006-2008

La distribución de las CSCA por los diagnósticos seleccionados mostró que $70 \%$ de las hospitalizaciones evitables se concentra en un número reducido de enfermedades, tabla 2. 
TABLA 2

Porcentaje de hospitalizaciones potencialmente prevenibles por diagnósticos de CSCA, hospitales seleccionados, Bogotá, 2006-2008

\begin{tabular}{|c|c|c|c|c|}
\hline \multirow[t]{2}{*}{ Enfermedades crónicas } & \multicolumn{4}{|c|}{ Porcentajes } \\
\hline & 2006 & 2007 & 2008 & Total periodo \\
\hline EPOC & 8.41 & 10.96 & 11.68 & 10.65 \\
\hline Diabetes & 6.90 & 6.06 & 6.22 & 6.32 \\
\hline Insuficiencia cardiaca & 5.78 & 6.10 & 6.55 & 6.21 \\
\hline Hipertensión arterial esencial & 5.26 & 6.15 & 6.64 & 6.14 \\
\hline Epilepsia & 5.45 & 4.02 & 4.00 & 4.35 \\
\hline Enfermedad cerebrovascular & 4.54 & 3.78 & 2.98 & 3.63 \\
\hline Asma sin especificar & 2.71 & 2.40 & 2.14 & 2.37 \\
\hline Infarto agudo del miocardio & 2.17 & 2.35 & 2.44 & 2.34 \\
\hline Fiebre reumática & 0.18 & 0.19 & 0.15 & 0.17 \\
\hline \multicolumn{5}{|l|}{ Cáncer } \\
\hline Cáncer de cuello uterino & 0.31 & 0.27 & 0.27 & 0.27 \\
\hline \multicolumn{5}{|l|}{$\begin{array}{l}\text { Enfermedades infecciosas y } \\
\text { parasitarias }\end{array}$} \\
\hline Bronquiolitis & 9.50 & 10.81 & 11.54 & 10.80 \\
\hline Enfermedad diarreica aguda & 9.00 & 10.61 & 9.61 & 9.83 \\
\hline Neumonía & 7.30 & 8.68 & 11.99 & 9.71 \\
\hline $\begin{array}{l}\text { Infección de vías urinarias sitio } \\
\text { no }\end{array}$ & 7.19 & 8.25 & 9.55 & 8,53 \\
\hline \multicolumn{5}{|l|}{ Especificado } \\
\hline Bronconeumonía & 8.62 & 5.31 & 4.67 & 5.83 \\
\hline Bronquitis aguda & 8.73 & 5.60 & 0.43 & 4.23 \\
\hline Celulitis & 2.62 & 1.66 & 2.79 & 2.35 \\
\hline $\begin{array}{l}\text { Rinofaringitis aguda (resfriado } \\
\text { común) }\end{array}$ & 1.34 & 1.12 & 1.19 & 1.20 \\
\hline Sífilis congénita & 0.74 & 0.80 & 0.69 & 0.74 \\
\hline Diarrea funcional & 0.00 & 1.23 & 0.60 & 0.68 \\
\hline Tuberculosis pulmonar & 0.49 & 0.71 & 0.57 & 0.60 \\
\hline Otitis media no supurativa & 0.50 & 0.54 & 0.66 & 0.58 \\
\hline Parasitosis intestinal & 0.29 & 0.29 & 0.29 & 0.29 \\
\hline \multicolumn{5}{|l|}{ Otras enfermedades } \\
\hline Gastritis & 1.55 & 1.61 & 1.75 & 1.65 \\
\hline Desnutrición proteico-calórica & 0.23 & 0.28 & 0.30 & 0.28 \\
\hline Anemia ferropénica & 0.19 & 0.22 & 0.28 & 0.24 \\
\hline Total CSCA & 19.35 & 21.64 & 24.43 & 22.05 \\
\hline
\end{tabular}

Fuente: RIPS de egresos hospitalarios 2006-2008 
Los grupos de edades que generan la mayoría de estas hospitalizaciones corresponden niños menores de 5 años y a adultos mayores de 60 . Para los niños: enfermedades agudas de las vías respiratorias altas y bajas, neumonías y bronquiolitis e infecciones del tracto urinario. Para los adultos mayores: enfermedades agudas y crónicas de las vías respiratorias bajas, hipertensión arterial, insuficiencia cardiaca, diabetes e infección urinaria.
El cálculo de los costos de hospitalización se hace mediante el producto de los días de estancia de los pacientes por la cantidad de egresos, en el estudio no fue posible hacer este cálculo debido a que sólo los hospitales Occidente de Kennedy, La victoria y Santa Clara entregaron los datos de las estancias y ninguno de los costos facturados. Diez enfermedades consumieron un total de 86.953 dias-estancias correspondientes a $14 \%$ del total de los egresos. La distribución por diez enfermedades de la lista de CSCA se muestra en la gráfica 3.

GRÁFICA 3

Días-estancia de diez CSCA en tres hospitales del estudio, Bogotá 2006-2008

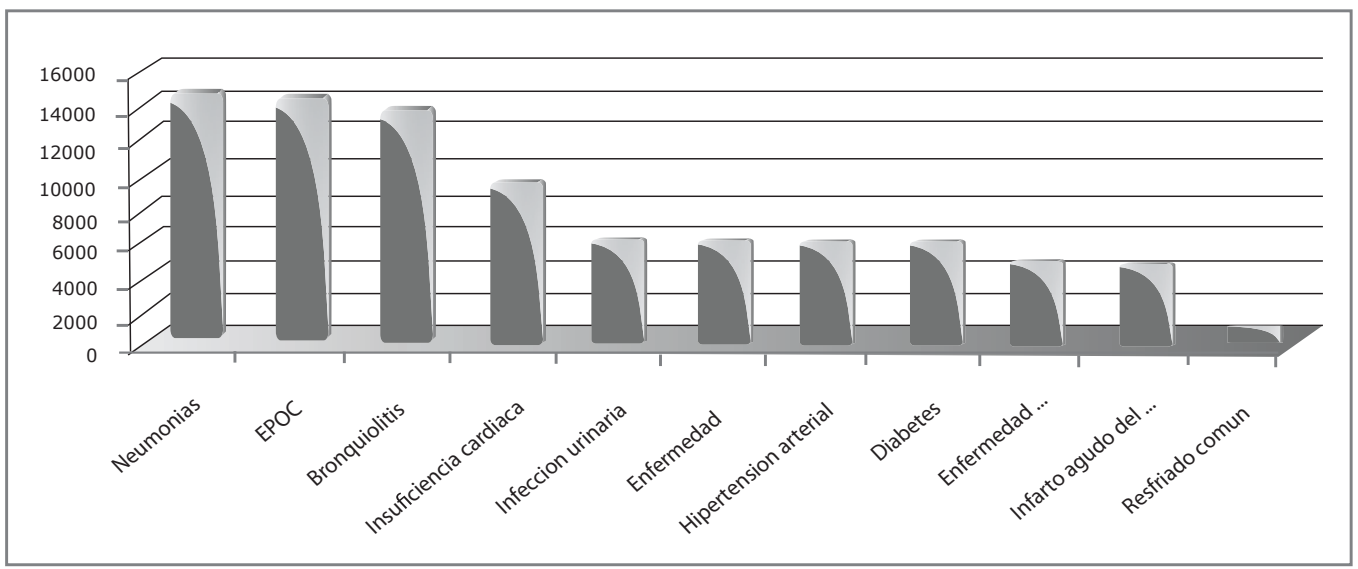




\section{Discusión}

El listado propuesto por diversos autores para identificar las hospitalizaciones sensibles a las intervenciones de APS, prueba su utilidad en este estudio piloto al facilitar la identificación de $22.5 \%$ de CSCA (14) (15).

Los diagnósticos encontrados en este estudio con base en el listado de CSCA contienen problemas de salud para las cuales la hospitalización no siempre es necesaria, como es el caso de la enfermedad diarreica aguda, el resfriado común, las celulitis o la fiebre reumática. En otros el ingreso puede estar condicionado al estado clínico del paciente, ejemplo la enfermedad pulmonar obstructiva crónica. Pero hay casos como la diabetes cuyo ingreso no está condicionado por las características del paciente.

Los resultados de este estudio sugieren que la mayoría de los problemas de salud identificados con base en el listado de CSCA, son susceptibles de ser atendidos con la atención primaria oportuna y de calidad que podría evitar o reducir sustancialmente su hospitalización. La capacidad de la APS para atender eficazmente a estas condiciones sensibles a su intervención, varíaenfunción del problema: diagnóstico precoz y tratamiento oportuno, programas continuos asistenciales (diabetes, hipertensión arterial, EPOC), actividades de prevención y promoción de estilos de vida saludable.
El porcentaje de CSCA encontrado en los egresos de los hospitales seleccionados pertenecientes a la Red Pública del Distrito Capital, podría estar influido por los estratos sociales que sirven estas IPS, que en su mayoría es población pobre no asegurada o perteneciente al régimen de seguridad social subsidiado. Esta población puede aportar el mayor porcentaje de hospitalizaciones evitables por su baja demanda de servicios de APS, por barreras económicas para acudir a tiempo al centro de salud primario, o por la mayor prevalencia de las enfermedades crónicas e infecciosas.

Este aspecto del estudio merece investigación posterior para aclarar si los pacientes hospitalizados por CSCA fueron atendidos en el nivel de atención primaria con antelación a su internación en los hospitales o sino, cuales son la barreras que les impiden su acceso oportuno a la APS, porque la tenencia de un carnet de afiliación o la promesa de cobertura de salud a los pobres no afiliados no garantiza su atención de salud oportuna.

Ahora bien, las diferencias en los porcentajes de CSCA entre los hospitales, también puede reflejar diferencias en las decisiones de la práctica médica frente a los problemas de salud.

Para estudios futuros se deberían ajustar los diagnósticos de CSCA, 
por grupos de expertos, para los tres grupos poblacionales de niños, adultos y ancianos.

Finalmente, este primer estudio sirve de inicio para continuar la evaluación de la capacidad de la APS para evitar las hospitalizaciones innecesarias de problemas de salud que podrían ser resueltos $o$ atendidos en el nivel de baja complejidad, y para poner en marcha acciones promuevan la capacidad resolutiva de este nivel.

\section{REFERENCIAS}

1. Sanderson C, Dixon J. (2000). Conditions for which onset hospital admission is potentialy preventable by timely and effective ambulatory care. J Health Service Policy, Vol. 5, págs. 222-30.

2. Brown AD, Goldacre MJ,Hiks N, Rourke JT, Murtry RY, Brown JD, Andersen Gm. (2001). Hospitalizationfor ambulatory care-sensitive conditions: ma methodfor comparative acces and quality studies using routinely collected satistics. Mar-Apr, Can J Public Health, Vol. 19, págs. 155-9.

3. Caminal J, Starfield B, Sánchez E, Casanova C, Morales M. (2004). The role ofprimary care in prevnetive ambulatory care sensitive conditions. Vol. 14, págs. 246-251.

4. FW., Porel. (2001). A comparison of ambulatory care-sensitive hospital discharge ratesfor Medicaid HMO enrollees and nonenrollees. Med Cares Res, Vol. 58, págs. 404-24.

5. FW., Porel. (2001). Avoidable hospitalizations and socioeconomic satatus in Galveston County, Texas. J Community Health, Vol. 19, págs. 377-387.

6. Blustein J, Hanson K. and Shea S. (1998). Preventable Hospitalizations ans socioeconomic status. Helath Affairs.

7. Márquez-Calderon S, Rodríguez del Águila M, Perca Milla E, Ortiz J Bermúdez Tamayo C. (2004). Características organizativas de la atención primaria y hospitalización por los principales ambulatory care sensitive conditions. Aten Primaria, Vol. 33, págs. 305-11. 
8. Ansari Z, Carson N, Serraglio A, Barbetti T, Cieutini F. (2002). The Victorian ambulatory care-sensitive conditions Study: Reducing demand on hospital servios in Victoria. Aust Health Rev, Vol. 25, págs. 71-7.

9. Falik M, Needleman J, Wells BI, Korb J. (2001). Ambulatory care-sensitive hospitalizations andemergency visits: esperiences of Medicaidpatients using federally qualified health centers. Med Care, Vol. 39, págs. 551-61.

10. Homar J. Caminal, Morales M. Espinosa, Ruiz E. Sánchez, Larrosa-CubellsM.J Bustins Poblet M. (2003). Hospitalizaciones prevenibles mediante una atención primaria oportuna y efectiva.Atención Primaria, Vol. 31, págs. 6-7.

11. Magan P., Otero Á. Alberquilla Á. and Ribera J. (2008). Geographic variations in avoidable hospitalizations in the edelry, in a health system with universal coverage. BMC Health Service Research, Vol. 18, págs. 8-42.

12.Caminal J, Márquez S y Sánchez E. (2001). Los Ambulatory Care-sensitive Conditions una medida de la capacidad resolutiva en España. Gac Sanit, Vol. 15, págs. 53-55.

13.Caminal J, Sánchez E, Morales M, Peiró R. y Márquez S. (2002). Avances en España en la investigación con el indicador "Hospitalización por Enfermedades Sensibles a Cuidados Ambulatorios”. Revista española de Salud Pública, Vol. 76.

14.ST., Fleming (1995). Primary care, avoidable hospitalization and outcomes ofcare: a literature review andmethodologicalaproach. Med Care, Vol. 52, págs. 88-108.

15.Billing J, Anderson GM, Newman LS. (1996). Recent fmding on preventable hospitalizations. Vol. 15, págs. 239-49. 